\title{
An application of a dynamic model of judgment to magnitude production
}

\author{
LAWRENCE T. DECARLO \\ Teachers College, Columbia University, New York, New York
}

\begin{abstract}
A dynamic model of judgment, together with a model of stimulus context effects, is applied to magnitude production (MP) and magnitude estimation (ME) experiments. Participants' responses in MP were correlated across trials, as is typically found for ME. The magnitude of the autocorrelation, however, was small, which suggests that participants in MP tend to rely more heavily on a long-term frame of reference. Second, a stimulus context effect found for ME did not appear for MP, most likely because of the different nature of the task (i.e., intermediate values of the stimulus were heard while the participant produced a response). A fit of an earlier regression model, on the other hand, suggests that the number presented on the previous trial in MP has a large contrastive effect on the current response. The present model offers a different view of this result, in that it shows that a negative coefficient for the earlier model is consistent with a positive judgmental effect. The regression effect noted by Stevens and Greenbaum (1966), which is a value of the estimated ME exponent that is smaller than the inverse of the estimated MP exponent, was also found; it is shown that the effect did not arise from bias in estimation.
\end{abstract}

In magnitude estimation (ME), stimuli are presented to participants and they are instructed to assign numbers proportional to their sensation magnitudes (see Gescheider, 1997; Stevens, 1986). Magnitude production(MP) reverses the situation in that numbers are presented and participants attempt to produce sensation magnitudes that are proportional to the numbers. It is well known that participants' responses in these types of tasks are not independent over trials, but rather are correlated. Although many studies have examined these sequential effects in ME experiments or related categorization and absolute identification experiments (e.g., Cross, 1973; DeCarlo, 1994; Garner, 1953; Holland \& Lockhead, 1968; Ward 1973, 1979; Ward \& Lockhead, 1970), few studies (to my knowledge) have examined sequential effects in MP, with the exception of a study by Green, Luce, and Duncan (1977) that is discussed below.

The present article shows that MP is useful for comparing and contrasting different models and theories of sequential effects. In particular, a dynamic model of judgment (DeCarlo, 1990) is applied MP. It is shown that the model provides insight into the structure of data obtained in ME and MP experiments and offers a simple account of similarities and differences found across the two procedures. The article begins with a brief review of the dynamic model as previously applied to ME, followed by an application of the model to MP.

\section{Dynamic Judgment}

A basic assumption in magnitude scaling experiments is that participants' judgments are proportional to their sen-

Correspondence should be addressed to L. T. DeCarlo, Department of Human Development, Box 118, Teachers College, Columbia University, 525 West 120th Street, New York, NY 10027 (e-mail: decarlo@exchange.tc.columbia.edu). sation magnitudes. There is, however, more than one way to make proportional judgments. One approach, for example, is to assign a reference response, say $R_{0}$ (i.e., an arbitrary modulus), to a reference sensation, say $\Psi_{0}$ (i.e., an arbitrary standard). On each trial, participants then compare their current sensation $\Psi_{t}$ to their reference sensation $\Psi_{0}$ and give a response $R_{t}$ so that the relation between $R_{t}$ and $R_{0}$ reflects the relation between $\Psi_{t}$ and $\Psi_{0}$,

$$
\frac{R_{t}}{R_{0}}=\frac{\Psi_{t}}{\Psi_{0}} v_{t}
$$

where $v_{t}$ represents random error. Rearranging gives

$$
R_{t}=\alpha_{0} \Psi_{t} v_{t}
$$

where the proportionality constant $\alpha_{0}=R_{0} / \Psi_{0}$. This shows that one way to make proportional judgments is to use a long-term frame of reference $\alpha_{0}$, which consists of an arbitrary response $R_{0}$ given to a reference sensation $\Psi_{0}$. Equation 1 represents the simple judgmental model of proportionality assumed by Stevens (1986); the basic assumption is that participants produce response relations, $R_{t} / R_{0}$, that approximate sensation relations, $\Psi_{t} / \Psi_{0}$. Equation 1 a shows that the assignment of an arbitrary response to a reference sensation in essence sets a unit of measurement $\alpha_{0}$ that is assumed to remain constant over time.

This approach is not the only way one can make proportional judgments. Another approach is to use the sensation and response from the previous trial as reference points. In this case, each sensation is compared with the previous sensation, and the participant gives a response that has the same relation to the previous response,

$$
\frac{R_{t}}{R_{t-1}}=\frac{\Psi_{t}}{\Psi_{t-1}} v_{t},
$$


which is essentially the idea underlying the response ratio hypothesis of Luce and Green (1974). The above can be rearranged as

$$
R_{t}=\alpha_{t-1} \Psi_{t} v_{t},
$$

from which it is apparent that all judgments are made relative to a short-term frame of reference, $\alpha_{t-1}=R_{t-1} / \Psi_{t-1}$; note that the proportionality constant in this case varies over time. To see how the error structure of Equation $2 \mathrm{a}$ differs from that of Equation 1a, substitute a lagged version of Equation 2a,

$$
R_{t-1}=\left(R_{t-2} / \Psi_{t-2}\right) \Psi_{t-1} v_{t-1},
$$

for $R_{t-1}$ in Equation 2 and rearrange, which gives

$$
R_{t}=\Psi_{t}\left(R_{t-2} / \Psi_{t-2}\right) v_{t} v_{t-1} .
$$

Repeating this procedure for $R_{t-2}, R_{t-3}$, and so on down to $R_{0}$ gives

$$
R_{t}=\alpha_{0} \Psi_{t} v_{t} v_{t-1} v_{t-2} \ldots v_{1},
$$

which shows that if the approach to the task shown by Equation 2 is used, then responses will again be proportional to sensation magnitudes, with proportionality constant $\alpha_{0}=R_{0} / \Psi_{0}$. Note, however, that the error structure of Equation $2 \mathrm{~b}$ differs from that of Equation 1a in that errors from previous trials (i.e., $v_{t-1}, v_{t-2}$ ) affect the current response in Equation 2b but not in Equation 1a.

Thus, there are at least two ways to make proportional judgments. The important aspect of Equations $1 \mathrm{a}$ and $2 \mathrm{~b}$ is that they show that the error structure provides information about the judgment process - if judgments are made relative to a stable reference, as in Equation 1, then the errors do not have effects extending over trials, whereas if judgments are made to a short-term reference, as in Equation 2, then the errors have effects extending over trials.

A dynamic model of relative judgment (DeCarlo, 1990; DeCarlo \& Cross, 1990) explicitly recognizes that there is more than one approach to the task. The idea of the model is that judgment is affected by both the shortand long-term frames of reference defined above, so that the frames are weighted as follows:

$$
R_{t}=\left(R_{0} / \Psi_{0}\right)^{1-\lambda} \Psi_{t}\left(R_{t-1} / \Psi_{t-1}\right)^{\lambda} v_{t},
$$

with $0 \leq \lambda \leq 1$. A basic idea of Equation 3 is that judgment is relative to both short- and long-term frames of reference. If $\lambda=0$, then all judgments are made relative to the long-term frame, $R_{0} / \Psi_{0}$, and Equation 3 reduces to Equation 1. If $\lambda=1$, then all judgments are made relative to the short-term frame, $R_{t-1} / \Psi_{t-1}$, and the model reduces to Equation 2. Values of $\lambda$ between zero and unity indicate that both frames of reference affect judgment, and in this way $\lambda$ provides a measure of the relative influence of the two reference frames. Equation 3, referred to here simply as the dynamic judgment model, can be viewed as a dynamic generalization of the classic model of proportional judgment of Stevens (1986) and the response ratio hypothesis of Luce and Green (1974).
The implications of Equation 3 for the error structure of magnitude estimates can be shown by using the approach noted above; that is, repeated substitution in Equation 3 for $R_{t-1}, R_{t-2}$, and so on down to $R_{0}$ gives

$$
R_{t}=\alpha_{0} \Psi_{t} v_{t}^{\lambda} v_{t-1}^{\lambda^{2}} v_{t-2}^{\lambda^{3}} \ldots v_{1}^{\lambda^{t-1}}
$$

which shows that errors from previous trials have a geometrically decaying influence on the current response. Another way to write the error process of Equation 4 is

$$
R_{t}=\alpha_{0} \Psi_{t} \epsilon_{t-1}^{\lambda} v_{t}
$$

where $\epsilon_{t}$ is nonrandom error (i.e., it is correlated with previous values of itself; that is, it is autocorrelated; for details, see DeCarlo, 1994; DeCarlo \& Cross, 1990). These equations can be transformed from a multiplicative to an additive relationship by taking logarithms, in which case the logarithm of the error process in Equation 5 gives a first-order autoregressive [AR(1)] error process, which is a basic type of process considered in time series analysis (e.g., Box \& Jenkins, 1976); note that the $\log$ arithm of the error process in Equation 4 gives the moving average representation of the AR(1) error process. Previous research (DeCarlo, 1990, 1992; 1994; DeCarlo \& Cross, 1990) has shown that, for ME and cross-modality matching experiments, the residuals obtained from a fit of a logarithmic transform of Stevens' power law are in fact well described by an $\mathrm{AR}(1)$ error process. ${ }^{1}$

In addition to providing a theoretical basis for $\mathrm{AR}(1)$ errors, the dynamic judgment model also suggests how to possibly gain some control over the autocorrelation. For example, if the instructions are varied so that they emphasize one reference frame over another, then the observed autocorrelation should be affected in a predictable way. In particular, the autocorrelation should be larger when the immediate frame of reference is emphasized, and this result has been found in several studies (DeCarlo, 1990, 1994; DeCarlo \& Cross, 1990). In addition, in an experiment by Ward (1987), larger autocorrelation was found with ratio ME instructions (which emphasize the previous trial) than with "absolute" ME instructions.

In summary, the dynamic judgment model provides a theoretical basis for the autocorrelation typically found in magnitude scaling experiments: Autocorrelation arises because of the influence of different frames of reference on judgment. Equation 3 shows that the autocorrelation parameter $\lambda$ provides a measure of the relative influence of the two frames of reference. The theory also suggests how the autocorrelation can be, in part, controlled.

\section{Stimulus Context Effects}

A large body of research has shown that prior stimulation appears to affect the current perception in magnitude scaling, categorization, and identification tasks (e.g., Cross, 1973; Garner, 1953; Holland \& Lockhead, 1968; Ward, 1973). One approach to modeling this stim- 
ulus context effect is to expand the power psychophysical model of Stevens (1986) to include an effect of the previous stimulus intensity, also via a power function,

$$
\Psi_{t}=S_{t}^{\beta} S_{t-1}^{\gamma} \delta_{t},
$$

where $\delta_{t}$ is random perceptual error. The parameter $\gamma$ reflects the magnitude of the effect of the previous stimulus intensity on the current perception and its sign indicates the direction of the effect. For example, Equation 6 with a positive value of $\gamma$ would indicate that prior stimulation had an additive effect on the current perception; note that Equation 6 with positive $\gamma$ also predicts a negative time-order error (see Hellström, 1985) in that if the same stimulus is presented twice in a row, it will be perceived as being greater in intensity on the second presentation. Cross (1973) introduced a variation of this where a positive value of $\gamma$ indicates an assimilative effect in that the perception of $S_{t}$ is larger when $S_{t-1}$ is greater than $S_{t}$ and is smaller when $S_{t-1}$ is less than $S_{t}$, and in this way the perception "assimilates" toward the previous stimulus intensity (whereas in the additive model of Equation 6, with a positive $\gamma$, the perception of $S_{t}$ is larger regardless of whether $S_{t-1}$ is greater than or less than $S_{t}$ ). It has previously been shown (DeCarlo \& Cross, 1990) that Cross's version of the model differs from Equation 6 only in that it replaces the exponent $\beta$ of $S_{t}$ with $\beta-\gamma$ (and so a positive value of $\gamma$ decreases the exponent).

Assuming that there are both judgment effects and stimulus context effects, in the sense of Equations 5 and 6 , substituting the latter into the former and taking logarithms gives

$$
\log R_{t}=\log \alpha_{0}+\beta \log S_{t}+\gamma \log S_{t-1}+\lambda e_{t-1}+u_{t},
$$

where $e_{t-1}=\log \epsilon_{t-1}$ and $u_{t}=\log v_{t}+\log \delta_{t}$. Equation 7 generalizes Stevens' regression model by including both a lagged regressor $\left(\log S_{t-1}\right)$ and an $\mathrm{AR}(1)$ error process $\left(\lambda e_{t-1}+u_{t}\right)$; it shows that a fit of the model provides direct estimates of the parameters $\gamma$ and $\lambda$, which reflect two types of context effects: an effect due to a judgmental process (i.e., the relativity of judgment, as indicated by $\lambda$ ) and an effect due to a perceptual or memory process (i.e., a stimulus context effect, as indicated by $\gamma$ ). Prior research has shown that the estimate of $\gamma$ for ME of loudness tends to be small and positive and, as noted above, the estimate of $\lambda$ is positive (DeCarlo, 1992, 1994; DeCarlo \& Cross, 1990).

The next section shows that the dynamic model of judgment (Equation 3), developed above for ME, and the stimulus context model (Equation 6) can both be immediately applied to MP.

\section{Dynamic Judgment and Stimulus Context in MP}

In MP, numbers are presented to participants and their task is to adjust a stimulus intensity so that their sensation magnitudes are proportional to the numbers. Thus, the responses are produced stimulus intensities and the stimuli are presented numbers, which is the reverse of ME.
The dynamic model of judgment can immediately be applied to MP. Note, for example, that one approach to the task is for participants to compare the current number $N_{t}$ to a reference number $N_{0}$ and to then produce a sensation magnitude $\Psi_{t}$ that stands in the same relation to a reference sensation $\Psi_{0}$,

$$
\frac{\Psi_{t}}{\Psi_{0}}=\frac{N_{t}}{N_{0}} v_{t} .
$$

For the short-term reference case, participants compare the current number to the previous number and produce a sensation magnitude that stands in the same relation to their previously produced sensation magnitude,

$$
\frac{\Psi_{t}}{\Psi_{t-1}}=\frac{N_{t}}{N_{t-1}} v_{t} .
$$

As before, if both frames of reference affect judgment, then the dynamic model of relative judgment is

$$
\Psi_{t}=\left(\Psi_{0} / N_{0}\right)^{1-\lambda} N_{t}\left(\Psi_{t-1} / N_{t-1}\right)^{\lambda} v_{t},
$$

which is simply Equation 3 rewritten for MP. This equation can also be reexpressed in the same manner as Equation 5, which gives

$$
\Psi_{t}=\left(1 / \alpha_{0}\right) N_{t} \epsilon_{t-1}^{\lambda} v_{t},
$$

where $\alpha_{0}=N_{0} / \Psi_{0}$ (as in ME, where $\alpha_{0}=R_{0} / \Psi_{0}$ ). Equation 8a shows that, if the above approach to judgment is used, then the produced sensation magnitudes are proportional to the presented numbers and the (log transformed) error process is again $\mathrm{AR}(1)$.

The model for stimulus context effects is the same as before-namely, Equation 6, which in this case allows for stimulus context effects of the prior (produced) stimulus intensity on the current sensation (it is assumed that numbers are perceived veridically). Substituting Equation 6 into Equation 8, solving for $S_{t}^{\prime}$ (where the prime is used to indicate that the stimulus intensity is produced by the participant) and taking logarithms gives

$$
\begin{aligned}
\log S_{t}^{\prime}= & (-1 / \beta) \log \alpha_{0}+(1 / \beta) \log N_{t} \\
& -(\gamma / \beta) \log S_{t-1}^{\prime}+(\lambda / \beta) e_{t-1}+(1 / \beta) u_{t},
\end{aligned}
$$

keeping in mind that the produced stimulus intensity $S_{t}^{\prime}$ is the response variable. Equation 9 has two basic implications. First, the error process is, as for ME, predicted to be AR(1) (note that the scaling constant $1 / \beta$ simply affects the variance of $u_{t}$ ). Second, Equation 9 shows that if $\gamma$ is positive, as found for ME, then the coefficient of the lagged produced stimulus intensity should be negative for MP. These predictions are examined next in a within-subjects design where each participant served in MP and ME experiments; the results are compared with those of a study by Green et al. (1977) where ME and MP conditions were also used (within subjects).

Equation 9 shows that the current $\log$ response $\left(\log S_{t}^{\prime}\right)$ should be regressed on the log of the current number $\left(\log N_{t}\right)$ and the $\log$ of the previous response $\left(\log S_{t-1}^{\prime}\right)$, 
allowing for an AR(1) error process. A difficulty arises with estimating the parameters of Equation 9 in the usual way, however, because of the combination of a lagged dependent variable $\left(\log S_{t-1}^{\prime}\right)$ on the right side of the equation together with an AR(1) error process (see the Appendix); the problem is well known in econometrics and is discussed in texts such as Greene (2000) and Johnston (1984). Fortunately, it is easily dealt with; maximum likelihood estimates of the model's parameters were obtained here using SAS (Release 8.02) employing the DEPVAR option in PROC AUTOREG (see the Appendix); the Appendix also notes an approach that can be implemented with standard software for regression.

\section{METHOD}

\section{Participants}

The participants were 8 students enrolled in an introductory psychology course. All participants claimed to have normal hearing. Each participant served in two sessions, which consisted of ME of loudness and MP of loudness to presented numbers. Four of the participants performed ME first, followed on a different day by MP; the other 4 performed MP first, followed on a different day by ME.

\section{Apparatus}

A General Radio Company oscillator was used to generate $1000-\mathrm{Hz}$ tones. The tones were presented binaurally through Grason Stadler headphones (TDH-39). The presentation of the stimuli and recording of the participant's responses were controlled by an IBM personal computer using a BASIC program written by L.T.D. Each participant was run one at a time in a sound-attenuating chamber (Industrial Acoustics Company) that contained headphones, a terminal, an intercom, and a KAT. The KAT is a pad with a surface that maps to the terminal; movements of a stylus or finger across the surface of the KAT moved an arrow on the terminal.

For ME, the order of presentation of the 12 stimuli, which ranged from 40 to $89.5 \mathrm{~dB}$ in $4.5-\mathrm{dB}$ steps, was determined by sequences of 120 trials generated by the uniform probability generator of SAS. The selected sequences had at least five presentations of each stimulus intensity. The autocorrelation function (ACF) and partial autocorrelation function (PACF) for each sequence were examined (for examples of the use of these functions in time series analysis, see Box \& Jenkins, 1976); the (six) selected sequences had no significant correlations for at least the first five lags. For MP, the stimulus to be adjusted was initially turned on at one of 50 values, ranging from 40 to $89 \mathrm{~dB}$; the initial value sequences were generated using the uniform probability generator of SAS and had no significant correlations for at least the first five lags of the ACF, and no significant cross-correlations with the stimulus presentation sequence it was paired with for at least the first five lags.

\section{Procedure}

Each participant served in two sessions that were separated by 1-4 days. The participants were first shown how to use the KAT. They were then required to practice using the KAT by entering five responses. For ME, the practice responses were numbers, whereas for MP, the practice responses were stimulus intensities adjusted by the participant.

Magnitude estimation. The participants were first given a practice session where they were required to make numerical estimates of eight line lengths, approximately 2, 3, 6, 12, 24, 48, and $192 \mathrm{~mm}$ in length, presented at least once each for a total of 20 trials. Ratio ME instructions were used: Participants were instructed to compare their current sensation with their previous sensation and produce a response that had the same relation (ratio) to their previous response.
Upon completion of the first practice session, participants were given a second practice session that consisted of 20 trials with the 12 stimuli (1000-Hz tones) used in the experiment; the stimuli ranged from $40 \mathrm{~dB}$ to $89.5 \mathrm{~dB}$ in steps of $4.5 \mathrm{~dB}$. Participants were told that the purpose was to familiarize them with the range of stimuli used in the experiment. The experiment, which consisted of 120 trials, then followed. On each trial, 1 of the 12 stimuli (same as in the second practice session) was presented for $1 \mathrm{sec}$. About $0.5 \mathrm{sec}$ after the offset of each tone, a numerical keypad appeared on the terminal; the keypad consisted of the numbers 0-9, a period (for decimals), and a clear entry key (to correct mistakes). Participants controlled the movement of an arrow on the terminal by moving a stylus or their finger across the surface of the KAT. Each digit of the chosen number could be entered by locating the arrow on a number and pressing a button on the KAT. The final response could be entered by moving the arrow to a box labeled "Enter" and pressing a button on the KAT. The next trial began $1 \mathrm{sec}$ after the response was entered.

Magnitude production. The participants were first given a practice session where they produced line lengths (using the KAT) in response to the numbers $2,3,5,7,10,15,20,30,50,75,125$, and 200; the numbers are similar to those used by Green et al. (1977), who noted that their choice of numbers followed in part from those typically given by participants. The 12 numbers were presented in a random order for a total of 20 trials. For the second practice session (20 trials), participants used the KAT to adjust the intensity of a $1000-\mathrm{Hz}$ tone in response to the 12 numbers listed above. Participants were instructed to compare each number to the previous number and to produce a loudness with the same ratio to the previous loudness (i.e., ratio MP instructions). On each trial, a number was presented for $2 \mathrm{sec}$ in the middle of the terminal; immediately after the number was presented, a $1000-\mathrm{Hz}$ tone was turned on at one of 50 random initial values ranging from 40 to $89 \mathrm{~dB}$. The participant could then adjust the intensity of the tone in $0.5-\mathrm{dB}$ steps by brushing a stylus or their finger across the surface of the KAT; movements to the right increased the intensity, whereas movements to the left decreased the intensity. It took about four strokes across the surface of the KAT to adjust the intensity across the full range, which was from 29 to $99 \mathrm{~dB}$. Participants entered their response by pressing a button on the KAT. The next trial began $1 \mathrm{sec}$ after participants entered their response. Following completion of the 20 practice trials, the experiment began. The experiment consisted of 120 trials and the procedure was the same as for the second practice session.

\section{RESULTS}

\section{Mean and Variability of Responses}

The left and right panels of Figure 1 present the medians (across participants) of the mean $\log$ responses to each $\log$ stimulus intensity (i.e., $\mathrm{dB} / 20$ ) for $\mathrm{ME}$ and to each log number for MP, respectively. In both cases, the trends are approximately linear.

\section{Time Series Analysis of Residuals}

The ACF and PACF function for the residuals obtained from maximum likelihood fits of the structural part of Equation 7 (ME) and Equation 9 (MP) were computed and plotted separately for each participant (the structural part involves only the regressors, and not the error process; see SAS/ETS user's guide, 1988). An AR(1) process implies a geometrically decaying ACF and a PACF with a drop after the first lag.

Figure 2 presents, for ME (upper panels) and MP (lower panels), the group ACF and PACF plots for the 

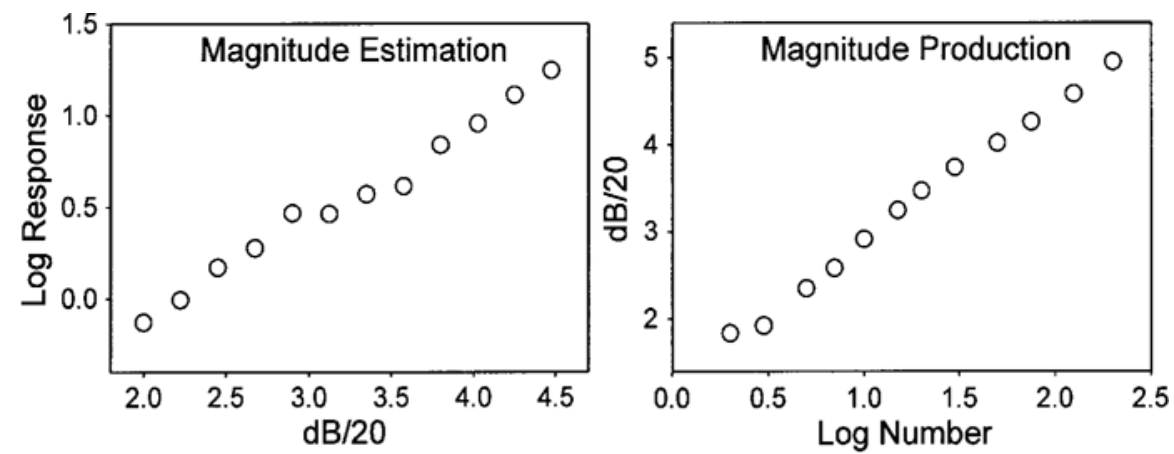

Figure 1. The left panel shows the mean log response in magnitude estimation as a function of the sound pressure level. The right panel shows the mean produced sound pressure level in magnitude production as a function of the log of the presented number.

residuals. The group plots were determined in two steps. First, the individual ACFs and PACFs were computed and plotted separately for each participant using PROC ARIMA of SAS (see SAS Institute, Inc., 1988). The medians and interquartile ranges of the correlations were then computed across participants for each lag and are shown in the figure. The group plots provide a summary of the individual plots (and the autocorrelations for individual participants are given in Table 1).

For ME, the ACF plot shows an approximate geometric decay and the PACF plot shows a drop after the first lag, which is consistent with an $\mathrm{AR}(1)$ error process.
This agrees with results found in previous studies for ME (e.g., DeCarlo, 1994; DeCarlo \& Cross, 1990). For MP, the ACF and PACF plots show that the correlations are small, and the plots are consistent with an AR(1) error process in that the PACF shows a drop after the first lag. The plots also show that the magnitudes of the correlations are smaller for MP than for ME.

\section{Regression Analysis}

Table 1 presents, for each participant, the results for fits of Equation 7 and Equation 9 for ME and MP, respectively (see the Appendix for details on how the mod-
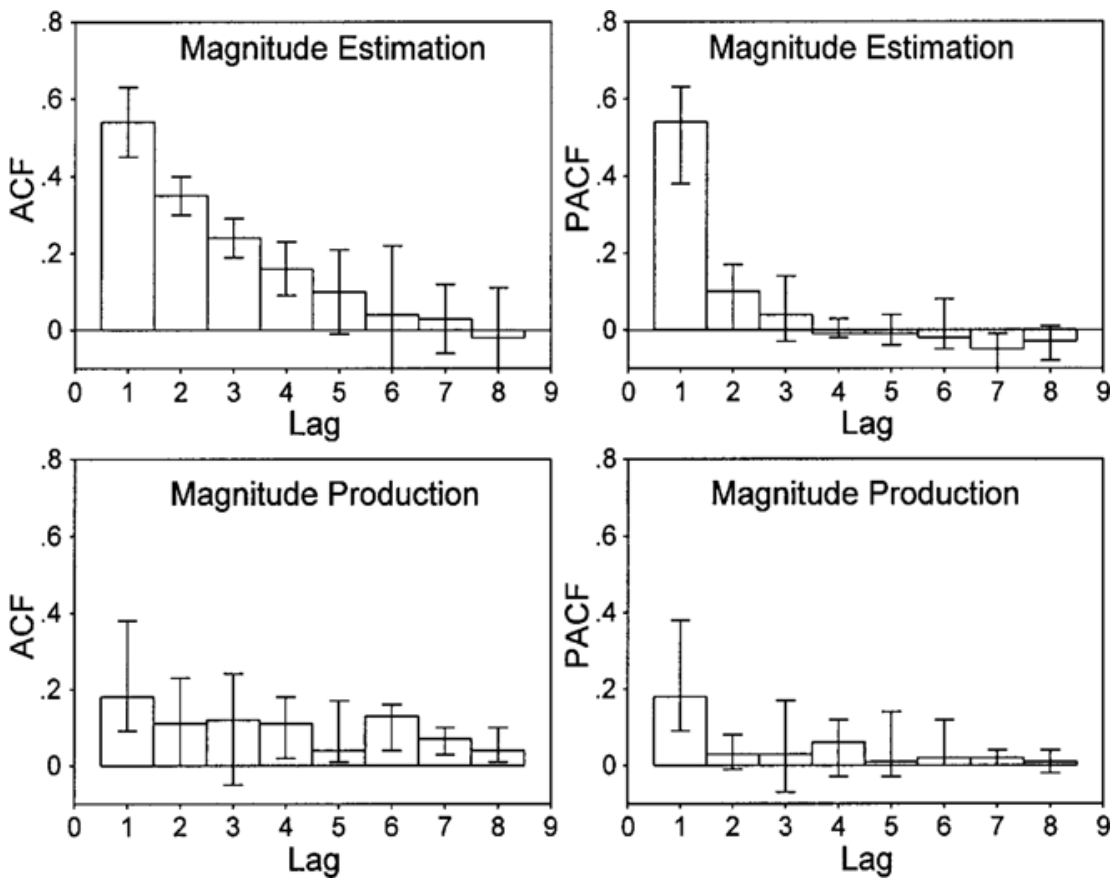

Figure 2. The upper left and right panels show, for magnitude estimation, the autocorrelation function and partial autocorrelation function, respectively, for the first eight lags. The lower left and right panels show, for magnitude production, the autocorrelation function and partial autocorrelation function, respectively, for the first eight lags. 
Table 1

Results for Each Participant for Magnitude Estimation (ME) and Magnitude Production (MP)

\begin{tabular}{|c|c|c|c|c|c|c|c|}
\hline \multirow[b]{3}{*}{ Participant } & \multicolumn{6}{|c|}{ Parameter Estimates for ME (Equation 7) } & \multirow[b]{3}{*}{$R^{2}$} \\
\hline & \multicolumn{2}{|c|}{$\beta$} & \multicolumn{2}{|c|}{$\gamma$} & \multicolumn{2}{|c|}{$\lambda$} & \\
\hline & Result & $S E$ & Result & $S E$ & Result & $S E$ & \\
\hline 1 & 0.36 & 0.01 & $0.05 * *$ & 0.01 & $.51 * *$ & 0.08 & .88 \\
\hline 2 & 0.50 & 0.02 & $0.06 * *$ & 0.01 & $.37 * *$ & 0.09 & .88 \\
\hline 3 & 0.75 & 0.06 & $0.22 * *$ & 0.06 & $.61 * *$ & 0.07 & 67 \\
\hline 4 & 0.56 & 0.03 & $0.12 * *$ & 0.03 & $.39 * *$ & 0.09 & .82 \\
\hline 5 & 0.55 & 0.03 & $0.10 * *$ & 0.03 & $.65^{* *}$ & 0.07 & .79 \\
\hline 6 & 0.56 & 0.03 & $0.09 * *$ & 0.03 & $.57 * *$ & 0.08 & .77 \\
\hline 7 & 0.45 & 0.02 & $0.11 * *$ & 0.02 & $.77 * *$ & 0.06 & .86 \\
\hline \multirow[t]{4}{*}{8} & 0.51 & 0.02 & $0.09 * *$ & 0.02 & $.39 * *$ & 0.09 & .89 \\
\hline & \multicolumn{6}{|c|}{ Parameter Estimates for MP (Equation 9) } & \\
\hline & \multicolumn{2}{|c|}{$1 / \beta$} & \multicolumn{2}{|c|}{$-\gamma / \beta$} & \multicolumn{2}{|c|}{$\lambda$} & \\
\hline & Result & $\overline{S E}$ & Result & $\overline{S E}$ & Result & $\overline{S E}$ & $R^{2}$ \\
\hline 1 & 1.79 & 0.07 & -0.06 & 0.03 & $.37 * *$ & 0.09 & .86 \\
\hline 2 & 1.66 & 0.07 & $-0.09 *$ & 0.04 & $.41 * *$ & 0.09 & .85 \\
\hline 3 & 1.21 & 0.06 & 0.08 & 0.05 & .05 & 0.11 & .78 \\
\hline 4 & 1.86 & 0.05 & -0.01 & 0.03 & .06 & 0.10 & .93 \\
\hline 5 & 1.73 & 0.07 & $-0.10 *$ & 0.04 & $.58 * *$ & 0.08 & .81 \\
\hline 6 & 1.04 & 0.05 & 0.04 & 0.05 & $.24 *$ & 0.10 & .81 \\
\hline 7 & 1.60 & 0.04 & 0.02 & 0.03 & .12 & 0.10 & .93 \\
\hline 8 & 1.63 & 0.04 & 0.04 & 0.02 & .11 & 0.10 & .93 \\
\hline
\end{tabular}

Note-Significance tests are not shown for the estimates of $\beta$ or $1 / \beta$ because all the estimates are significant (i.e., several times larger than their standard errors). $* p<.05 . \quad * * p<.01$.

els were fit). The values of $R^{2}$ for both ME and MP, which are generally about .80 or higher, show that the data are well described by Equations 7 (for ME) and 9 (for MP) for each individual (the reported values of $R^{2}$ are for both the structural and error parts of the model, referred to as the total $R^{2}$ in SAS; see the SAS/ETS user's guide, 1988).

For ME, the maximum likelihood estimates of $\beta$ are around 0.5 . The estimates of $\gamma$ are positive and are significantly different than zero for all 8 participants. The estimates of the autocorrelation parameter $\lambda$ are all positive and significant, and are similar in magnitude to those found in previous studies (where ratio ME instructions were used). For MP, the estimates of $1 / \beta$ are all greater than unity, as typically found (Participant 6's value is close to unity). The estimates of $-\gamma / \beta$ are negative for 4 participants and positive for the other 4 participants, but are small in magnitude and only two (which are negative) are significant. The estimates of $\lambda$ are positive, but are significant for only 4 of the 8 participants.

\section{DISCUSSION}

With respect to ME, the results are similar to those obtained in prior studies (see Table 2 of DeCarlo \& Cross, 1990): The estimates of the autocorrelation were positive and the estimates of the coefficient of the lagged log stimulus intensity were small and positive. In terms of the dynamic judgment model, these results can be interpreted as showing, respectively, that (1) judgment was affected by both short-term and long-term frames of ref- erence, which were weighted about equally; and (2) the prior stimulus had a small additive effect on the perception of the current stimulus.

With respect to MP, positive autocorrelation was found, but it was smaller in magnitude than that found for ME (in spite of the fact that participants in both the MP and ME experiments were told to use the previous response and sensation as the reference). This is an interesting result in that it suggests that the short-term frame might be weighted less heavily in MP than in ME; a similar result appears in a study of Green et al. (1977), as discussed below. Second, the estimates of the coefficient of the lagged log response were close to zero and significant for only 2 of the 8 participants. The two significant estimates were negative, which is consistent with Equation 9 and indicates a positive effect of the prior stimulus, since the coefficient provides an estimate of $-\gamma / \beta$. Thus, the results for MP are consistent with those found for ME of loudness, in that the estimates of $\gamma$ are positive (for nonzero values), but they also differ in that the estimates are clearly close to zero for MP. The implication is that MP differs from ME in that the stimulus context effect is smaller or nonexistent. This seems reasonable because, for ME, one value of each stimulus was heard for $1 \mathrm{sec}$ on each trial, whereas for MP, several values of each produced stimulus were heard on each trial while the participant adjusted the stimulus; this might reduce or eliminate the effect of the previously produced stimulus.

The next section compares the present results to those of Green et al. (1977), who presented estimates for a different regression model. In order to compare the results, relations between the alternative model and the models considered here are derived.

\section{Jesteadt et al.'s Regression Model}

Jesteadt, Luce, and Green (1977) proposed the following regression model to study sequential effects:

$$
\begin{aligned}
\log R_{t}= & \alpha_{0}+\alpha_{1} \log S_{t}+\alpha_{2} \log S_{t-1} \\
& +\alpha_{3} \log R_{t-1}+u_{t} .
\end{aligned}
$$

The results found with this model, as applied to ME, are discussed first, followed by an illustration of its application to MP.

Magnitude estimation. It has previously been shown that the estimates of $\alpha_{1}$ and $\alpha_{3}$ obtained for fits of Equation 10 are close to those obtained for $\beta$ and $\lambda$ in Equation 7. The estimates of $\alpha_{2}$, on the other hand, tend to be large and negative, which is in contrast to the small positive values of the estimates of $\gamma$ found for Equation 7 . The finding of a negative $\alpha_{2}$ for fits of Jesteadt et al.'s (1977) model has led some researchers to conclude that the effect of the previous stimulus intensity is contrastive for loudness estimation. The dynamic judgment model, however, accounts for the contradictory results in that it shows that a positive value of $\gamma$ in Equation 7 can be consistent with a negative value of $\alpha_{2}$ in Jesteadt et al.'s model (see DeCarlo, 1992, 1994; DeCarlo \& Cross, 
1990). This can be seen by substituting the stimulus context model (Equation 6) into the dynamic judgment model (Equation 3 ) and taking logarithms, which gives

$$
\begin{aligned}
\log R_{t}= & (1-\lambda) \log \alpha_{0}+\beta \log S_{t}+(\gamma-\lambda \beta) \log S_{t-1} \\
& +\lambda \log R_{t-1}-\lambda \gamma \log S_{t-2}+u_{t} .
\end{aligned}
$$

Note that omitting $\log S_{t-2}$ from the above results in a model with the same regressors as Jesteadt et al.'s model. Although Equation 11 differs from Equation 10 in that it includes $\log S_{t-2}$, it has previously been shown (e.g., DeCarlo \& Cross, 1990) that it allows one to closely predict the coefficients obtained for fits of Equation 7 from a fit of Equation 10 (and vice versa), as will also be shown below.

Equation 11 provides an interpretation of the coefficients of Jesteadt et al.'s (1977) model (Equation 10) in terms of the dynamic judgment model (Equation 3) and the stimulus context model (Equation 6), which is really the only purpose for deriving it. In particular, it suggests that the estimate of $\alpha_{2}$ for a fit of Jesteadt et al.'s model is an estimate of $\gamma-\lambda \beta$. It follows that $\alpha_{2}$ will be negative if $\gamma<\lambda \beta$, which appears to be the typical case for loudness estimation. Thus, according to the dynamic judgment model, negative values of $\alpha_{2}$ are found for loudness estimation with Jesteadt et al.'s model because the coefficient of the lagged log stimulus regressor confounds perceptual (i.e., $\gamma$ and $\beta$ ) and judgment (i.e., $\lambda$ ) effects, and not because there is a contrastive effect of the prior stimulus. This view is supported by several results found in prior research, as discussed by DeCarlo (1992, 1994) and DeCarlo and Cross (1990).

The upper half of Table 2 presents the mean coefficients obtained for ME in the present study and those obtained for ME (of loudness) in a study by Green et al. (1977), who reported results for ME and MP in a withinsubjects design. Green et al. provided the coefficients obtained for a fit of Jesteadt et al.'s (1977) model (Equation 10), and so the parameters of Equation 7 can be estimated (using Equation 11). Table 2 shows the results. With respect to the dynamic judgment model, the left side of the table shows that the mean coefficient of the lagged $\log$ stimulus for Equation 7 was small and positive for both studies and the autocorrelation was large and positive. The right side of the table shows that the results for Equation 10 differ in that the mean coefficient of the lagged log stimulus was large and negative in both the present study and that of Green et al. This shows yet again that a positive estimate of the coefficient of the lagged log stimulus for Equation 7 can be consistent with a negative estimate for Equation 10. Note that the results are consistent with Equation 11; for example, using Equation 11 and the results of the present study, the coefficient of the lagged log stimulus for Equation 10 predicted from a fit of Equation 7 is $0.10-0.53 \times$ $0.53=-0.18$, which is reasonably close to the estimate of -0.13 obtained by fitting Equation 10 .

Thus, the upper half of Table 2 shows that the results of the present study for ME are consistent with those ob-
Table 2

Mean Estimates for Magnitude Estimation (ME) and Magnitude Production (MP) for the Current Experiment

\begin{tabular}{|c|c|c|c|c|c|c|}
\hline \multirow[b]{3}{*}{ Study } & \multicolumn{6}{|c|}{ Magnitude Estimation } \\
\hline & \multicolumn{3}{|c|}{ Equation 7} & \multicolumn{3}{|c|}{ Equation 10} \\
\hline & $\beta$ & $\gamma$ & $\lambda$ & $\alpha_{1}$ & $\alpha_{2}$ & $\alpha_{3}$ \\
\hline Current & 0.53 & 0.10 & 0.53 & 0.54 & -0.13 & 0.50 \\
\hline \multirow[t]{4}{*}{ GLD 1977} & 0.44 & $0.06^{\mathrm{a}}$ & 0.53 & $0.44^{b}$ & $-0.17^{b}$ & 0.53 \\
\hline & \multicolumn{6}{|c|}{ Magnitude Production } \\
\hline & \multicolumn{3}{|c|}{ Equation 9} & \multicolumn{3}{|c|}{ Equation 10} \\
\hline & $1 / \beta$ & $-\gamma / \beta$ & $\lambda$ & $\alpha_{1}$ & $\alpha_{2}$ & $\alpha_{3}$ \\
\hline Curre & 1.56 & -0.01 & 0.23 & 1.55 & -0.43 & 0.24 \\
\hline GLD 1977 & 1.53 & $-0.02^{\mathrm{c}}$ & 0.27 & $1.53^{\mathrm{d}}$ & $-0.44^{\mathrm{d}}$ & 0.27 \\
\hline
\end{tabular}
and that of Green, Luce, and Duncan (1977)

Note-GLD 1977 is Green, Luce, and Duncan (1977). $\quad$ aThe value of $\gamma$ for Equation 7 was estimated from the results reported for Equation 10 as $\left(\alpha_{1} \times \alpha_{3}\right)+\alpha_{2}$ (see Equation 11). bThe coefficients are twice as big as those reported by GLD because they used sound power $(\mathrm{dB} / 10)$ whereas sound pressure $(\mathrm{dB} / 20)$ was used here; using sound power simply has the effect of halving the estimates of $\alpha_{1}$ and $\alpha_{2}\left(\alpha_{3}\right.$ is not affected). 'The value of $-\gamma / \beta$ for Equation 9 was estimated from the results reported for Equation 10 as $\left(\alpha_{2} / \alpha_{1}\right)+\alpha_{3}$ (see Equation 12). dThe coefficients are one half as big as those reported by GLD because they used sound power $(\mathrm{dB} / 10)$ whereas sound pressure $(\mathrm{dB} / 20)$ was used here; using sound power simply has the effect of doubling the estimates of $\alpha_{1}$ and $\alpha_{2}\left(\alpha_{3}\right.$ is not affected).

tained by Green et al. (1977). The table shows that the negative values found for the coefficient of the lagged $\log$ stimulus intensity in Jesteadt et al.'s (1977) model for ME of loudness are consistent with a positive stimulus context effect in the dynamic judgment model, as shown by the positive estimates of $\gamma$ for fits of Equation 7 .

Magnitude production. The implications of the dynamic judgment model for Equation 10 can be shown by substituting Equation 6 into Equation 8 and taking logarithms, which gives

$$
\begin{aligned}
\log S_{t}^{\prime}= & (1 / \beta) \log N_{t}-(\lambda / \beta) \log N_{t-1} \\
& +[\lambda-(\gamma / \beta)] \log S_{t-1}^{\prime} \\
& +(\lambda \gamma / \beta) \log S_{t-2}^{\prime}+(1 / \beta) u_{t},
\end{aligned}
$$

where the intercept, $[(\lambda-1) / \beta] \log \alpha_{0}$, has been omitted for clarity. Again, omitting $\log S_{t-2}^{\prime}$ from the results above in a model with the same regressors as Jesteadt et al.'s (1977) model, and in this way Equation 12 provides an interpretation of the coefficients of Jesteadt et al.'s model in terms of the dynamic judgment model, as applied to MP; specifically, $\alpha_{1}=1 / \beta, \alpha_{2}=-\lambda / \beta$, and $\alpha_{3}=\lambda-(\gamma / \beta)$ (note that a direct fit of Equation 10 will give estimates that differ to some extent from those predicted by Equation 12 due to the omission of $\log S_{t-2}^{\prime}$ ). It is shown next that Equation 12 is useful for estimating the coefficients of Equation 9 from a fit of Equation 10 (of course, one should fit Equation 9 directly; Equation 12 is used here because only estimates for a fit of Equation 10 were available for Green et al.'s, 1977, study).

The lower half of Table 2 presents, for MP, the mean coefficients for both the present study and that of Green et al. (1977). The table shows that the results are again 
consistent across the two studies. With respect to the dynamic judgment model, the left side of the table shows that the estimate of $-\gamma / \beta$ is negative and near zero for both studies. Note that the estimate of $\gamma$ obtained by dividing $\gamma / \beta$ by the estimate of $1 / \beta$ is essentially zero; as noted above, this suggests that the stimulus context effect in MP is near zero, most likely because of the different nature of the task (i.e., participants must pass through and hear other stimulus intensities before arriving at their final response). Second, the autocorrelation (the estimate of $\lambda$ ) is positive for both studies and smaller than that found for ME; the implication is that participants in MP tend to rely more heavily on a long-term frame of reference, regardless of the instructions.

With respect to Jesteadt et al.'s (1977) model, the right side of the table shows that the major difference is that the estimate of the coefficient of the lagged log number $\left(\alpha_{2}\right)$ is large and negative for both the present study and that of Green et al. (1977); it is also larger in magnitude than the estimate of the coefficient of the lagged log stimulus intensity obtained for ME. In the absence of theory, this would suggest a large contrastive effect of the previous number on the perception of the current number; the finding of a larger estimate for MP suggests that the stimulus context effect for numbers is larger than that for loudness. In my view, a contrast effect for numbers does not seem likely (although some might argue for a contrast effect for numbers); moreover, the finding that the effect is larger for numbers than for loudness seems even less likely. In any case, the dynamic judgment model offers a different view of these results. Specifically, Equation 12 shows that the coefficient of the lagged log number in Jesteadt et al.'s model, $\alpha_{2}$, is an estimate of $-\lambda / \beta$. Thus, in terms of the dynamic judgment model, the large negative values of $\alpha_{2}$ found for fits of Jesteadt et al.'s model to MP data arise because the coefficient reflects a judgment effect (i.e., the autocorrelation $\lambda$ ), and not because there is a large contrastive effect of the previous number.

In summary, the results for Green et al.'s (1977) within-subjects ME and MP experiments are quite consistent with the results found here. The present experiments and those of Green et al. together support the view that the coefficients of Equations 7 and 9 provide simple measures of judgment and perceptual processes in magnitude scaling tasks. The models also account for results obtained for fits of Jesteadt et al.'s model that would otherwise be puzzling, such as the large negative value of the coefficient of the lagged log number in MP.

Prior research has shown that the dynamic judgment model provides a simple account of other puzzling results found with Jesteadt et al.'s (1977) model. For example, it has been shown that using ME instructions that emphasize the short-term frame over the long-term frame leads to a considerable increase in autocorrelation $(\lambda)$, as expected, and also to an increase in (negative) $\alpha_{2}$ in Jesteadt et al.'s model, which is not expected (DeCarlo, 1990, 1994; DeCarlo \& Cross, 1990). Equation 11 shows that this result is consistent with the parameter constraint $\alpha_{2}=\gamma-\lambda \beta$ : An increase in $\lambda$ should be accompanied by an increase in (negative) $\gamma-\lambda \beta$ (keeping in mind that $\alpha_{2}$ is negative because $\lambda \beta$ is larger than positive $\gamma$ ). Second, whereas the estimates of $\gamma$ for fits of Equation 7 were smaller with longer intertrial intervals (ITIs), the results for fits of Jesteadt et al.'s model were the opposite: The estimates of $\alpha_{2}$ were negative and larger for longer ITIs (see DeCarlo, 1992). This result is counterintuitive in that it is expected that the magnitude of the contrast should be smaller when the previous stimulus is further removed in time and/or space. Equation 11, however, again sheds light on this result: If $\alpha_{2}=$ $\gamma-\lambda \beta$, then a decrease in positive $\gamma$ will lead to an increase in negative $\alpha_{2}$ (again keeping in mind that $\gamma<\lambda \beta$ ), and a fit of Equation 7 showed that the estimate of $\gamma$ was indeed smaller for the longer ITI. Thus, the dynamic judgment model combined with the stimulus context model together provide a simple account of several results found with Jesteadt et al.'s model; see DeCarlo $(1992,1994)$ and DeCarlo and Cross (1990) for further discussion. The results found here for MP add to this evidence.

\section{Some Notes on Other Results}

Positive and negative context effects. Although the results discussed here suggest a positive stimulus context effect for loudness estimation, this is not meant to imply that, in general, the context effect is always positive (this simply appears to be the case for several continua, as shown by DeCarlo, 1994, DeCarlo \& Cross, 1990, and Schifferstein and Frijters, 1992). For example, Schifferstein and Frijters presented results that suggested a negative stimulus context effect for sweetness estimation. Moreover, it is important to note that a negative effect for sweetness was found both for a fit of Jesteadt et al.'s (1977) model (Equation 10) and for a fit of the model with autocorrelated errors (Equation 7), as was also noted by Schifferstein and Frijters (p. 252). This is consistent with the relation between the parameters of the models discussed above. That is, if $\gamma$ is negative (indicating a contrast effect), then $\alpha_{2}=\gamma-\lambda \beta$ will be negative (for positive $\lambda$ and $\beta$, as is usually found). Thus, the study of Schifferstein and Frijters provides evidence of a contrast effect for sweetness estimation, with the direction of the effect remaining the same regardless of which model is used (which is the crucial finding). On the other hand, loudness estimation offers a different example where a (possibly) positive context effect appears to be negative if a different model is used.

The inverted-V and spatial-temporal errors. Some other results found in studies of sequential effects have also been noted; for details and a review of studies up until the late 1990s, see Baird (1997). Here I comment on a result referred to as the inverted-V (or triangle) pattern. In particular, Jesteadt et al. (1977) noted that, in addition to successive responses being correlated, there was a pattern to the correlations (note that this has been shown only for data pooled across participants, and not for in- 
dividual data). Specifically, the pattern was that the correlations were higher when successive stimuli were close in value and smaller when successive stimuli were farther apart. This suggests that a model with simple autocorrelated errors might be incomplete. DeCarlo and Cross (1990, p. 387) commented on this finding and noted that the relative judgment model could be extended to include the effect; they suggested using a dummy coded variable that took on values of zero or one depending on the distance of successive stimuli, which is consistent with the idea of an attention band, as discussed by Luce and Green (1978). The resulting model is an extension of the dynamic judgment model with time series errors (Equation 7) to a model with spatial-temporal errors. Estimation of the model is more involved, and the model has not been investigated to date. DeCarlo and Cross (1990) noted that the extension offered a simple interpretation of the inverted-V pattern-namely, that the short-term frame is used when successive stimuli are similar and the long-term frame is used when they are far apart, but they also noted that the importance and psychological relevance of the extension remains to be demonstrated.

The inverted-V pattern can be viewed as a secondorder effect; it suggests that the simple model with autocorrelated errors might be incomplete. In a similar vein, Stevens' regression model is incomplete because it does not account for correlated errors. Note, however, that this does not mean that Stevens' model must be abandoned, but rather that it should be extended to recognize the correlation; this is accomplished by the dynamic judgment model, which extends the model and provides a theoretical basis for autocorrelated errors. Similarly, the inverted-V pattern suggests possibly further extending the dynamic judgment model to allow for a pattern of correlations, which leads to models with spatial-temporal errors, as discussed above. Note that the usual result of ignoring autocorrelation or spatial autocorrelation is that the stan- dard errors are incorrect, so that significance tests cannot be trusted. The parameter estimates are still unbiased, however, which is important because the focus in research on sequential effects has been on mean parameter estimates (as in Table 2). This means, for example, that conclusions about the size of the stimulus context effect across different conditions (e.g., different ITIs) are likely still valid even if there is a spatial aspect of the autocorrelations that is ignored. As another example, the inverted-V graphs presented in DeCarlo and Cross (1990) showed that the correlations were larger for the condition where the short-term frame of reference was emphasized, so once again the conclusions are the same: The instructions can be varied so as to affect the magnitude of the autocorrelation. The point is that a possible second-order pattern in the errors does not necessarily invalidate results found with the basic time series regression model.

Figure 3 presents a graph of the correlations of the (structural) residuals as a function of the difference between successive stimulus magnitudes (see the Appendix in DeCarlo \& Cross, 1990, for notes on computational details). The left panel shows that an inverted-V pattern appears for ME, whereas the right panel suggests that the pattern might also appear for MP, although it is less clear and the interquartile ranges (indicated by the brackets) show that there is considerable variability across participants. The graph also shows that, relative to ME, the correlations for MP tend to be smaller. So again, conclusions about differences in autocorrelation across ME and MP are the same as for the regression analysis-namely, that the correlation appears to be smaller for MP than for ME.

\section{Assimilation and the Regression Effect}

The "regression effect" discussed by Stevens and Greenbaum (1966) refers to the finding that the exponent obtained for ME is smaller than the inverse of the
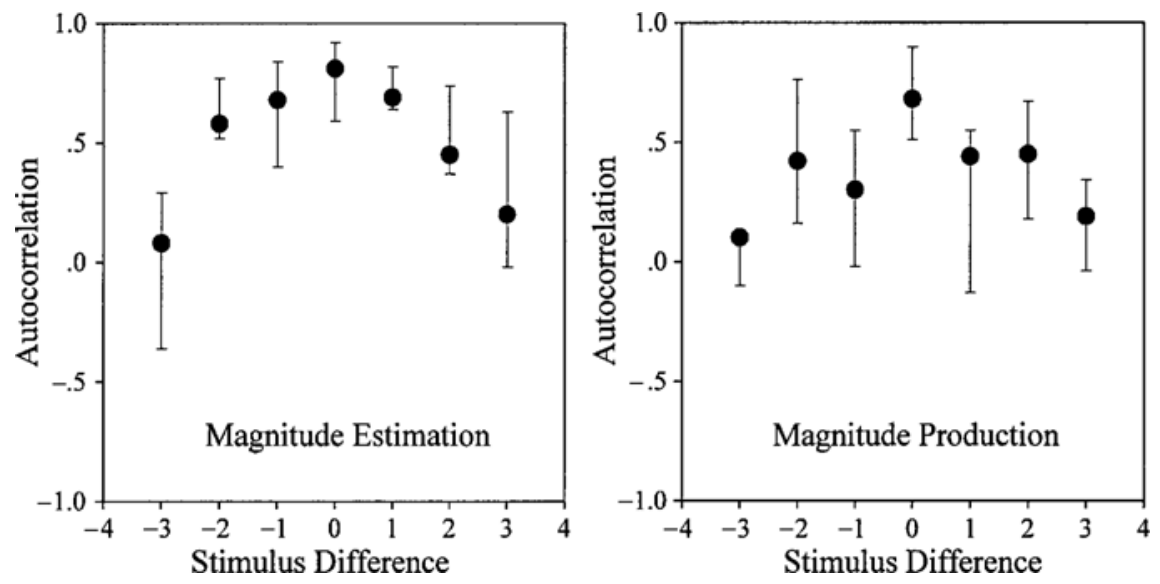

Figure 3. The left panel shows, for magnitude estimation, the correlation of successive structural residuals computed separately for each categorical stimulus difference. The right panel shows the same for magnitude production. The categorical stimulus differences for the stimuli used here are $0=0 \mathrm{~dB}, 1=4.5-13.5 \mathrm{~dB}$ difference, $2=18-27 \mathrm{~dB}$ difference, and $3=31.5-49.5 \mathrm{~dB}$ difference. 
MP exponent. This was also found here: The estimate of $\beta$ obtained for ME was 0.53 , and the estimate of $\beta$ obtained for MP by taking the inverse is $1 / 1.56=0.64$; similarly, for Green et al.'s (1977) study, the estimate of $\beta$ for ME was 0.44 and the estimate of $\beta$ for MP is $1 / 1.53=$ $0.65 .^{2}$ Thomas (1981), however, noted that the estimate of $\beta$ obtained by taking the inverse of the estimate of $1 / \beta$ obtained for MP (i.e., with Equation 9) is biased. The estimate can be approximately corrected for the bias (see Equation 10 of Thomas, 1981). With respect to the present study, the correction factor (which is subtracted from the estimate of $1 / \beta$ ) is very small-the correction factor is $\hat{\sigma}^{2} /\left(a^{3} \times d\right)$, where $\hat{\sigma}^{2}$ is the estimate of the residual variance, $a$ is the estimate of $1 / \beta$ obtained for MP (from Equation 9), and $d$ is the sum of the squared deviations for the predictor. For the 8 participants in the present study, the largest value of $\hat{\sigma}^{2}$ was 0.22 , the smallest value of $a^{3}$ was 1.12 , and $d$ was larger than 20 , so the correction is less than 0.01 . Thus, the correction is too small to account for the difference of 0.11 between 0.64 and 0.53 , so the regression effect found here is not due to statistical bias; Thomas reported results for matches of brightness to line length and line length to brightness that gave similar results in that the bias was again too small to account for the regression effect.

Another possible explanation of the regression effect was suggested by Cross (1973). As noted, in Cross's version of the model, the exponent $\beta$ in Equation 6 is replaced by $\beta-\gamma$. An interesting implication of this version of the model is that if the size of the stimulus context effect $(\gamma)$ in MP is less than that found for ME, then a "regression effect" would result. That is, the estimate of $\beta-\gamma$ obtained for ME would be smaller than the

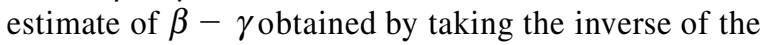
coefficient of $\log N_{t}$ for MP if $\gamma$ was larger for ME than for MP. As shown above, the estimate of $\gamma$ was indeed larger for ME than for MP in both the present study and that of Jesteadt et al. (1977). Note that for the present study, the difference between the mean estimates of $\beta$ across ME and MP is $0.64-0.53=0.11$, whereas for Green et al.'s (1977) study, the difference is $0.65-0.44=$ 0.21 ; the difference between the mean estimates of $\gamma \mathrm{ob}-$ tained across ME and MP is less than 0.10 for both studies. Thus, it is interesting that assimilation could account for part (but not all) of the regression effect found in the present study, but its magnitude is clearly too small to account for the regression effect found in Green et al.'s study. Further research on the regression effect is needed.

\section{Summary}

According to the dynamic judgment model, the correlation over trials of participants' responses in ME and MP experiments arises from the comparative nature of the task and the influence of different frames of reference on judgment. The model makes specific predictions about the dynamic structure of data obtained with ME and MP. It is shown that the parameter estimates obtained for ME and MP were consistent across the two procedures: The estimate of the autocorrelation was positive, albeit smaller for MP than for ME, and the estimate of $\gamma$ was positive, but close to zero for MP. These results are only apparent, though, when regression models suggested by the theory presented here are used; an earlier regression model gives different results. The experiments show that there are invariant aspects of sequential effects across ME and MP, and the dynamic model of relative judgment provides a simple interpretation of the results.

\section{REFERENCES}

BAIRD, J. C. (1997). Sensation and judgment: Complementarity theory of psychophysics. Mahwah, NJ: Erlbaum.

Box, G. E. P., \& Jenkins, G. M. (1976). Time series analysis: Forecasting and control (rev. ed.). San Francisco: Holden-Day.

Cross, D. V. (1973). Sequential dependencies and regression in psychophysical judgments. Perception \& Psychophysics, 14, 547-552.

DeCarlo, L. T. (1990). Sequential effects in magnitude scaling (Doctoral dissertation, SUNY at Stony Brook, 1989). Dissertation Abstracts International, 50, 3730B.

DeCarlo, L. T. (1992). Intertrial interval and sequential effects in magnitude scaling. Journal of Experimental Psychology: Human Perception \& Performance, 18, 1080-1088.

DeCARLO, L. T. (1994). A dynamic theory of proportional judgment: Context and judgment of length, heaviness, and roughness. Journal of Experimental Psychology: Human Perception \& Performance, 20, 372-381.

DeCarlo, L. T., \& Cross, D. V. (1990). Sequential effects in magnitude scaling: Models and theory. Journal of Experimental Psychology: General, 119, 375-396.

GARNer, W. R. (1953). An informational analysis of absolute judgments of loudness. Journal of Experimental Psychology, 46, 373-380.

Gescheider, G. A. (1997). Psychophysics: The fundamentals (3rd ed.). Hillsdale, NJ: Erlbaum.

Green, D. M., Luce, R. D., \& Duncan, J. E. (1977). Variability and sequential effects in magnitude production and estimation of auditory intensity. Perception \& Psychophysics, 22, 450-456.

Greene, W. H. (2000). Econometric analysis (4th ed.). Upper Saddle River, NJ: Prentice-Hall.

Hellst RöM, Å. (1985). The time-order error and its relatives: Mirrors of cognitive processes in comparing. Psychological Bulletin, 97, 35-61.

Holland, M. K., \& LockHEAD, G. R. (1968). Sequential effects in absolute judgments of loudness. Perception \& Psychophysics, 3, 409-414.

Jesteadt, W., Luce, R. D., \& GreEn, D. M. (1977). Sequential effects in judgments of loudness. Journal of Experimental Psychology: Human Perception \& Performance, 3, 92-104.

Johnston, J. (1984). Econometric methods (2nd ed.). New York: McGraw-Hill.

LuCE, R. D., \& GREEN, D. M. (1974). The response ratio hypothesis for magnitude estimation. Journal of Mathematical Psychology, 11, 1-14.

LucE, R. D., \& Green, D. M. (1978). Two tests of a neural attention hypothesis for auditory psychophysics. Perception \& Psychophysics, 23, 363-371.

SAS INSTITUTE, INC. (1988). SAS/ETS user's guide (Version 6, 1st ed.). Cary, NC: Author.

Schifferstein, H. N. J., \& Frijters, J. E. R. (1992). Contextual and sequential effects on judgments of sweetness intensity. Perception \& Psychophysics, 52, 243-255.

Stevens, S. S. (1986). Psychophysics. New Brunswick, NJ: Transaction Books.

Stevens, S. S., \& Greenbaum, H. B. (1966). Regression effect in psychophysical judgment. Perception \& Psychophysics, 1, 439-446.

Thomas, H. (1981). Estimation in the power law. Psychometrika, 46, 29-34.

WARD, L. M. (1973). Repeated magnitude estimations with a variable standard: Sequential effects and other properties. Perception \& Psychophysics, 14, 193-200. 
WARD, L. M. (1979). Stimulus information and sequential dependencies in magnitude estimation and cross-modality matching. Journal of Experimental Psychology: Human Perception \& Performance, $\mathbf{5}$, 444-459.

WARD, L. M. (1987). Remembrance of sounds past: Memory and psychophysical scaling. Journal of Experimental Psychology: Human Perception \& Performance, 13, 216-227.

WARD, L. M., \& LoCKHEAD, G. R. (1970). Sequential effects and memory in category judgments. Journal of Experimental Psychology, $\mathbf{8 4}$ 27-34.

\section{NOTES}

1. Of course, the model can be readily extended to allow for influences of frames further back than the first trial, which results in a higher order autoregressive error process, but prior research suggests that effects from further trials back are at most very small, as also shown by the PACF plots in Figure 2.

2. With respect to the individual data, note that 6 of the participants clearly showed a regression effect, whereas Participant 4 did not, and the effect was small for Participant 5.

\section{APPENDIX}

Some Notes on Estimation

\section{Magnitude Estimation}

It can be shown that it follows from Equation 7 (i.e., see Equation 11) that

$$
\begin{aligned}
\log R_{t}-\lambda \log R_{t-1}= & (1-\lambda) \log \alpha_{0}+\beta\left(\log S_{t}-\lambda \log S_{t-1}\right) \\
& +\gamma\left(\log S_{t-1}-\lambda \log S_{t-2}\right)+u_{t},
\end{aligned}
$$

which shows that, given an estimate of $\lambda$, the variables can be transformed as $\log R_{t}-\lambda \log R_{t-1}, \log S_{t}-\lambda$ $\log S_{t-1}$, and $\log S_{t-1}-\lambda \log S_{t-2}$, and the resulting error, $u_{t}$, is uncorrelated. Thus, given an estimate of $\lambda$, ordinary regression can be performed on the transformed variables to obtain estimates of $\beta$ and $\gamma$. This suggests a two-step approach, which can easily be implemented in standard software for regression. The first step is to obtain a consistent estimate of $\lambda$ by using the residuals obtained from a regression of $\log R_{t}$ on $\log S_{t}$ and $\log S_{t-1}$; this estimate can then be used to transform the variables as shown above, and the second step is to perform regression on the transformed variables. This is the basis of various two-stage estimation procedures (e.g., see Johnston, 1984). More efficient estimates can be obtained using PROC AUTOREG of SAS, as done for the results presented here (using maximum likelihood).

\section{Magnitude Production}

Equation 9 implies (using Equation 12) that

$$
\begin{aligned}
\log S_{t}^{\prime}-\lambda \log S_{t-1}^{\prime}= & (1 / \beta)\left(\log N_{t}-\lambda \log N_{t-1}\right) \\
& -(\gamma / \beta)\left(\log S_{t-1}^{\prime}-\lambda \log S_{t-2}^{\prime}\right) \\
& +(1 / \beta) u_{t},
\end{aligned}
$$

where the intercept, $[(\lambda-1) / \beta] \log \alpha_{0}$, has been omitted for clarity. This shows that the error term for the transformed variables is uncorrelated. In this case, however, one cannot obtain an estimate of $\lambda$ by using the residuals obtained from a regression of $\log S_{t}^{\prime}$ on $\log N_{t}$ and $\log S_{t-1}^{\prime}$ because the estimate will be inconsistent (because of the presence of a lagged dependent variable on the right side of the equation). Several approaches to this problem have been discussed (e.g., see Johnston, 1984). A simple approach is to use a grid search (starting, e.g., with steps of 0.1 ) over the possible values of $\lambda$, which are between -1 and 1 . For each value of $\lambda$, the variables are transformed as shown above, and the value of $\lambda$ that minimizes the sum of the squared residuals is taken as the estimate of $\lambda$. A finer grid can then be used (e.g., steps of 0.01 around the initial estimate of $\lambda$ ). The final step is to use the estimate of $\lambda$ to transform the variables and to run a regression with the transformed variables. The resulting parameter estimates will be consistent (and unbiased), but the standard errors need to be corrected, as shown by Johnston (1984). For the results reported here, maximum likelihood estimates were obtained using PROC AUTOREG of SAS (and the LAGDEP option; see SAS/ETS user's guide, 1988). 